\title{
Chronic burden of near-roadway traffic pollution in 10 European cities (APHEKOM network]
}

\author{
Laura Perez ${ }^{1,2}$, Christophe Declercq ${ }^{3}$, Carmen Iñiguez ${ }^{4,5}$, Inmaculada Aguilera ${ }^{5,6}$, \\ Chiara Badaloni ${ }^{7}$, Ferran Ballester ${ }^{4,5}$, Catherine Bouland ${ }^{8}$, Olivier Chanel $^{9}$, \\ Francisco B. Cirarda ${ }^{10}$, Francesco Forastiere ${ }^{7}$, Bertil Forsberg ${ }^{11}$, \\ Daniela Haluza ${ }^{12}$, Britta Hedlund ${ }^{13}$, Koldo Cambra ${ }^{14}$, Marina Lacasaña ${ }^{5,15}$, \\ Hanns Moshammer ${ }^{12}$, Peter Otorepec ${ }^{16}$, Miguel Rodríguez-Barranco ${ }^{15}$, \\ Sylvia Medina ${ }^{3}$ and Nino Künzli $i^{1,2}$
}

Affiliations: 'Swiss Tropical and Public Health Institute, Basel, and ${ }^{2}$ University of Basel, Basel, Switzerland. ${ }^{3}$ Institut de Veille Sanitaire, Paris, and ${ }^{9}$ Aix Marseille University (Aix-Marseille School of Economics), CNRS \& EHESS, Marseille, France. "University of Valencia, Center for Public Health Research (CSISP), Valencia, ${ }^{5}$ Spanish Consortium for Research on Epidemiology and Public Health (CIBERESP). ${ }^{6}$ Centre for Research in Environmental Epidemiology (CREAL), Hospital del Mar Research Institute (IMIM), Barcelona, ${ }^{10}$ Dirección Territorial de Sanidad de Bizkaia, Bilbao, ${ }^{14}$ Centro de Investigación Biomédica, Pamplona, and ${ }^{15}$ Escuela Andaluza de Salud Pública (EASP), Spain. ${ }^{7}$ Dept of Epidemiology, Lazio Regional Health Service, Rome, Italy. ${ }^{8}$ Brussels Institute for the Management of the Environment, Brussels-Capital Region, School of Public Health, Université Libre de Bruxelles - ULB, Brussels, Belgium. ${ }^{11}$ Occupational and Environmental Medicine, Umeå University, Umeå, and ${ }^{13}$ Swedish Protection Agency, Stockholm, Sweden. ${ }^{12}$ Medical University of Vienna, Austria, Institute of Environmental Health, Center for Public Health, Vienna, Austria. ${ }^{16}$ National Institute of Public Health, Ljubljana, Slovenia.

Correspondence: L. Perez, Swiss Tropical and Public Health Institute, Socinstrasse 57, P.0. Box, 4002, Basel$\mathrm{CH}$, Switzerland. E-mail: I.perezQunibas.ch

ABSTRACT Recent epidemiological research suggests that near road traffic-related pollution may cause chronic disease, as well as exacerbation of related pathologies, implying that the entire "chronic disease progression" should be attributed to air pollution, no matter what the proximate cause was. We estimated the burden of childhood asthma attributable to air pollution in 10 European cities by calculating the number of cases of 1) asthma caused by near road traffic-related pollution, and 2) acute asthma events related to urban air pollution levels. We then expanded our approach to include coronary heart diseases in adults.

Derivation of attributable cases required combining concentration-response function between exposures and the respective health outcome of interest (obtained from published literature), an estimate of the distribution of selected exposures in the target population, and information about the frequency of the assessed morbidities.

Exposure to roads with high vehicle traffic, a proxy for near road traffic-related pollution, accounted for $14 \%$ of all asthma cases. When a causal relationship between near road traffic-related pollution and asthma is assumed, $15 \%$ of all episodes of asthma symptoms were attributable to air pollution. Without this assumption, only 2\% of asthma symptoms were attributable to air pollution. Similar patterns were found for coronary heart diseases in older adults.

Pollutants along busy roads are responsible for a large and preventable share of chronic disease and related acute exacerbations in European urban areas.

@ERSpublications

APHEKOM network: pollution near busy roads is a large, preventable source of chronic disease and acute episode triggers http://ow.ly/mkpRH

Received: Feb 222012 | Accepted after revision: Dec 282012 | First published online: March 212013

Support statement: APHEKOM project (Improving Knowledge and Communication for Decision Making on Air Pollution and Health in Europe) was funded jointly by the European Commission's Programme on Community Action in the Field of Public Health (2003-2008) under grant agreement no. 2007105.

Conflict of interest: None declared.

Copyright @ERS 2013 


\section{Introduction}

Health impact assessments of air pollution usually rely on the availability of relevant epidemiological studies $[1,2]$. In recent years, substantial progress has been made in unravelling the effects of air pollution on health. Two findings in particular have led to a revision of approaches used to derive attributable burden.

First, numerous studies indicate that air pollution contributes to the development of chronic pathologies, and thus affects the incidence and prevalence of chronic diseases [3-5]. In the past, the chronic effects of air pollution have been taken into account by estimating the number of life years lost due to long-term exposure to air pollution. The morbidity burden is considered only for acute effects of air pollution (e.g. cardiorespiratory hospitalisations, bronchitis symptoms, myocardial infarctions or restricted activity days) $[1,6,7]$. However, chronic morbidity due to air pollution also heavily impacts health and the healthcare system in addition to acute effects. Thus, the burden of chronic disease due to air pollution has been grossly underestimated.

Secondly, recent evidence shows that living in close proximity to busy roads has a negative impact on health [5]. Near-road concentrations of traffic-related gases and particles, in particular the ultrafine fraction of particles, are many times higher than urban background levels along busy roads [5]. This spatial pattern is not observed for the pollutants most often investigated in impact assessments, such as PM10, PM2.5 (particulate matter up to $10 \mu \mathrm{m}$ or $2.5 \mu \mathrm{m}$ in size) or ozone. Furthermore, experimental studies show that the health effects of ultra-fine near-road particles are not necessarily the same as the effects of larger particles [8]. Therefore, common measurement strategies and health assessments may not adequately characterise near-road pollution and associated health impacts.

Few epidemiological studies use the near-road pollutants for exposure assessment. The studies which do examine near-road pollutants, including several European studies, suggest that the spatial distribution of near-road traffic-related pollutants corresponds to the distribution of a variety of health effects [9-16]. Of special concern is the role that near-road exposure may play in the development of chronic pathologies, such as asthma and cardiovascular diseases, including coronary heart disease (CHD) [3-5]. Evidence linking near-road pollution exposure to childhood asthma, the most prevalent chronic disease in children, is particularly strong $[3,4]$. Children living close to busy roads are more likely to develop asthma [3]. In contrast, markers of secondary particulate pollution, such as PM2.5 or PM10, are not so clearly associated with childhood asthma incidence or prevalence [3]. This indicates that a more refined technique is needed to evaluate exposure and related health effects, and/or there are different mechanisms involved in the toxicity of near-road pollutants compared to urban background particulate matter.

If a risk factor, e.g. air pollution, affects both the development of chronic pathologies and the exacerbation of acute events, usual methods to derive the burden of events attributable to the risk factor become inappropriate [17]. For chronic diseases caused by a particular risk factor, the entire "disease progression", including acute events, should be attributed to the risk factor, regardless of the proximate cause [18]. Traditional air pollution impact assessments quantified only the burden of acute events directly associated with exposure. A recent case study of two communities in Southern California (USA) revealed that traditional methods greatly underestimated the impact of air pollution on childhood asthma [17, 19]. This effect may be particularly strong in Europe, where there are high urban densities, increased traffic loads, and limited policies which address the concentrations of near-road pollutants, such as ultrafine particles. Therefore, there is strong need to update methods and expand health impact assessments in Europe.

Taking into account recent findings, the objective of this study was to determine the burden of acute events related to the chronic "morbidity progression" attributable to near-road traffic-related pollution, compared to the burden of acute events attributable to urban background levels of air pollution in 10 European cities. While our assessment focused on childhood asthma, we also expanded our analysis to include chronic conditions in adults, in particular CHD.

\section{Material and methods}

We used population-attributable fractions (PAFs) to estimate the health impact of air pollution in 10 European cities, all of which are partners in the APHEKOM project: improving knowledge and communication for decision making on air pollution and health in Europe. PAF is the standard methodology used to assess the contribution of a factor to disease. It uses excess risks obtained from epidemiological studies and applies them to target populations. This assessment is based on methods developed previously [17]. Derivation of attributable cases required knowledge about the concentrationresponse function (CRF) between exposures and the respective health outcome of interest (obtained from published literature), an estimate of the distribution of selected exposures in the target population, and 
information about the frequency of the assessed morbidities. Specific assumptions and the data used are presented below. We identified CRFs for the association between near-road traffic-related pollution and chronic outcomes based on a recent review [5]. Residential proximity to busy roads, defined as $>10000$ vehicles per day, was selected as a marker of long-term exposure to near-road traffic-related pollution (tables 1 and 2). Consistent with our recent studies [19], the Californian study was used for childhood asthma data.

For the acute outcomes, we obtained CRFs from hospitalisation and symptom studies (table 1 and 2). The acute effect evaluation was limited to the impact of ambient concentrations of PM10 or $\mathrm{NO}_{2}$. Meta-analytic estimates were derived using inverse-variance-weighting when several studies were available. We corrected the odds ratio, calculated from the odds ratios of published studies, to avoid overestimating the true relative risks in our final estimations [24]. Our target population was restricted to children aged 0-17 years for asthmarelated outcomes and to adults older than 65 years for CHD-related outcomes. Hospital data were obtained from local registries. Other morbidity prevalence data that could not be drawn from local surveys were extrapolated from studies used to derive the CRFs (table 3).

A near-road buffer to define "exposed populations to near-road traffic-related pollution" was determined based on the studies used to derive the CRFs: $75 \mathrm{~m}$ for asthma and $150 \mathrm{~m}$ for CHD. In the absence of routinely available distributions of populations along roads, methods developed previously were applied in all cities [17]. In brief, data from population distribution maps given at centroids of census blocks were distributed within a hypothetical grid across residential areas to represent the number of inhabitants per household. Traffic density maps, showing roads with more than 10000 vehicles per day (busy roads), were then overlaid and the distance from each grid point to the nearest pre-identified busy road was calculated to derive the population living within the distance-based near-road buffers. The level of detail available to develop these distributions varied across cities. As a rule, the most detailed information was always used. For example, population data given at the door address of buildings was preferred over assuming a distribution of population over larger geographical units (i.e. when data was available at block level only). For cities for which complete traffic counts or traffic models were not available, local expertise and/or urban planning information was used to select major traffic arteries. In the case of exposure to urban background

\section{TABLE 1 Concentration-response functions (CRFs) used in the evaluation for asthma and related acute morbidities}

$\begin{array}{lcccc}\text { Outcome } & \text { Unit of CRF } & \begin{array}{c}\text { Location, age of } \\ \text { study participants }\end{array} & \begin{array}{c}\text { Definition of outcome } \\ \text { and/or frequency outcome } \\ \text { in study population }\end{array} & \begin{array}{c}\text { CRF } \\ \left(95 \% \mathrm{Cl}^{\#}\right.\end{array}\end{array}$

\section{Prevalence of underlying chronic diseases}

Asthma prevalence

Residence $\leqslant 75 \mathrm{~m}$ of
busy roads defined as
freeways, other high-
ways, and arterial
roads
USA,
Prevalence exposure
$\quad 15 \%$

PM10 per $10 \mu \mathrm{g} \cdot \mathrm{m}^{-3} \quad$ Meta-analytic review, children

$\begin{array}{cc}\text { PM10 per } 5 \mu \mathrm{g} \cdot \mathrm{m}^{-3} & \text { Copenhagen, } \\ & \text { Denmark, } \\ & 5-18 \text { years } \\ \text { PM10 per } 10 \mu \mathrm{g} \cdot \mathrm{m}^{-3} & \text { APHEA study, } \\ & \text { Europe, } \\ & 0-14 \text { years }\end{array}$

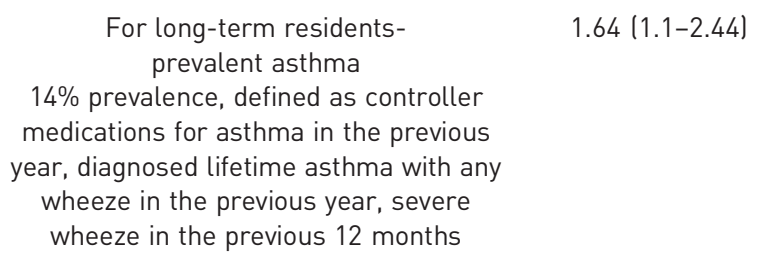

$1.028(1.016-1.039)$

ICD10: J45, 46

ICD9: $490-496$

$1.07(1.00-1.15)$

(transformed from

PM2.5)

$1.012(1.001-1.023)$

[23]

\section{Meta-analytic estimate, $\mathrm{PM} 10$ per $1 \mu \mathrm{g} \cdot \mathrm{m}^{-3}$}

PM10: particulate matter up to $10 \mu \mathrm{m}$; ICD: international classification of diseases; PM2.5: particulate matter up to $2.5 \mu \mathrm{m}$; APHEA: Air pollution and health, a European approach. \#: when estimates were reported as odds ratios, odds ratios were corrected with the formula CRF/(1+It(CRF-1)), where It is estimated as the frequency of the outcome in the population [24]. 
TABLE 2 Concentration-response functions (CRFs) used in the evaluation in relation to coronary heart disease and related acute morbidity

\begin{tabular}{|c|c|c|c|c|c|}
\hline Outcome & Unit of CRF & $\begin{array}{c}\text { Location, age of study } \\
\text { participants }\end{array}$ & $\begin{array}{l}\text { Definition of outcome and/or } \\
\text { frequency outcome in study } \\
\text { population }\end{array}$ & $\operatorname{CRF}(95 \% \mathrm{Cl})^{\#}$ & Study \\
\hline
\end{tabular}

\section{Prevalence of underlying chronic diseases CHD prevalence}

\section{Exacerbations}

Non-fatal MI

Hospital admission for MI

Hospital admission for stroke
Busy road $\leqslant 150 \mathrm{~m}$ from home ldefined as autobahn and federal highways: 10-

$110 \times 10^{3}$ vehicles per dayl

Prevalence exposure $31.4 \%$

$\mathrm{PM} 2.5$ per $10 \mu \mathrm{g} \cdot \mathrm{m}^{-3}$
North-Rhine-Westphalia, Germany, 45-74 years

Ausburg 1999-2001, $>18$ years
Rome 1995-1997, $>18$ years

5 European studies, $>35$ years

London 1987-1994, all ages

$$
\begin{aligned}
& \text { PM10 per } 12 \mu \mathrm{g} \cdot \mathrm{m}^{-3} \\
& \text { PM10 per } 10 \mu \mathrm{g} \cdot \mathrm{m}^{-3} \\
& \text { PM10 per } 10 \mu \mathrm{g} \cdot \mathrm{m}^{-3} \\
& \text { PM10 per } 25 \mu \mathrm{g} \cdot \mathrm{m}^{-3}
\end{aligned}
$$

Helsinki 1998-2004, $\geqslant 65$ years

9 French cities, $\geqslant 65$ years

APHEA, 8 European cities, all ages Southwestern Sweden, median age 70 years
$7.1 \%$, prevalence, defined as self-reported history of MI or application of a coronary stent or angioplasty or bypass surgery

WHO algorithm (includes evaluation chest pain, $Q$ waves examination, levels creatine kinase and other)

Transformed to PM10 per $1 \mu \mathrm{g} \cdot \mathrm{m}^{-3}$ ICD9-410

ICD9: 410

ICD9:410

Meta-analytic estimate, $\mathrm{NO}_{2}$ per $1 \mu \mathrm{g} \cdot \mathrm{m}^{-3}$

ICD10: 160-61 and 163-164

ICD10: 160-I64; G45-G46

ICD9: $430-8$

Ischaemic stroke

Meta-analytic estimate, PM10 per $1 \mu \mathrm{g} \cdot \mathrm{m}^{-3}$
$1.85(1.21-2.84)$

1.0007

(0.9993-1.0020)

CHD: coronary heart disease; MI: myocardial infarction; PM2.5: particulate matter up to $2.5 \mu \mathrm{m}$; PM10: particulate matter up to $10 \mu \mathrm{m}$; WHO: World Health Organization; ICD: international classification of diseases; APHEA: Air pollution and health, a European approach. \# : when estimates were reported as odds ratios, odds ratios were corrected with the formula CRF/(1+It(CRF-1)), where It is estimated as the frequency of the outcome in the population [24].

pollution, used for the acute exacerbation estimates, annual average concentrations derived from urban background fixed site monitors of PM10 and $\mathrm{NO}_{2}$ were assumed to apply to the entire population. Daily levels measured at the fixed site monitors were used to calculate annual average concentrations using standard city protocols.

The disease burden attributable to air pollution strongly depends on the points of reference, which in turn may depend on policy questions. In the case of the disease burden from near-road traffic-related pollution, we provided only one scenario: the total burden of these chronic conditions attributable to living within the determined buffers. In other words, the implicit assumption is that if no one lived within these buffers, the burden would be prevented. Alternatively, this translates into a scenario in which those living along busy roads were no more exposed to traffic-related pollutants than those living outside of the buffer zone. If all near-road effects were due to tail pipe emissions, the latter scenario would reflect cities with only zeroemission vehicles on the roads.

In the case of urban background pollution, we used an annual mean of $20 \mu \mathrm{g} \cdot \mathrm{m}^{-3}$ as the scenario reference point. For PM10, this reflects the World Health Organization (WHO) guideline value [34]. For both PM10 and $\mathrm{NO}_{2}$, the scenario reference point reflects an annual mean not yet reached in any of the participating 


\begin{tabular}{|c|c|c|c|c|c|c|c|c|c|c|c|c|}
\hline 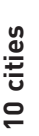 & & 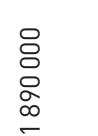 & $\stackrel{\infty}{\stackrel{\sim}{\simeq}}$ & $\begin{array}{l}0 \\
\infty \\
\infty \\
m\end{array}$ & $\stackrel{\cong}{\cong}$ & & $\begin{array}{l}\stackrel{\circ}{\circ} \\
\circ \\
\stackrel{\circ}{\infty} \\
-\end{array}$ & $\bar{\pi}$ & $\stackrel{\llcorner}{\sim}$ & $\begin{array}{l}\bar{\alpha} \\
\stackrel{\alpha}{L} \\
\stackrel{L}{L}\end{array}$ & $\begin{array}{l}\text { তั } \\
\stackrel{\circ}{\circ} \\
\llcorner\end{array}$ & 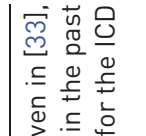 \\
\hline 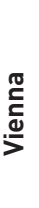 & & 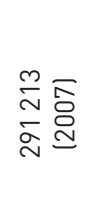 & $\stackrel{\infty}{\stackrel{\infty}{\llcorner}}$ & $\bar{m}$ & 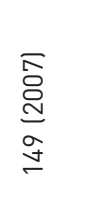 & & 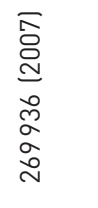 & 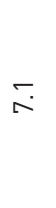 & 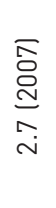 & 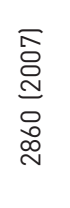 & 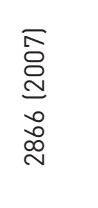 & 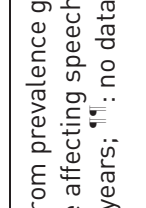 \\
\hline 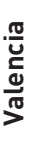 & & 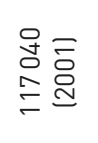 & $\stackrel{\circ}{=}$ & $\begin{array}{l}\text { L } \\
\text { లో }\end{array}$ & 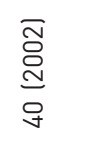 & & 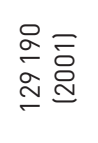 & $\check{\tau}$ & $\stackrel{\sim}{\sim}$ & 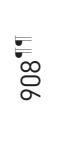 & 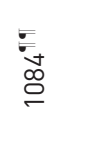 & 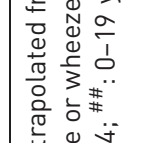 \\
\hline 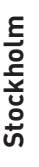 & & 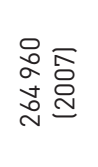 & $\stackrel{m}{a}$ & 品 & 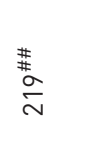 & & 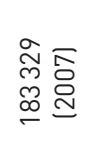 & $\check{\sim}$ & $\stackrel{\sim}{\sim}$ & $\begin{array}{l}\overline{\bar{亠}} \\
\text { 品 }\end{array}$ & 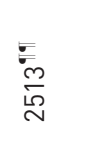 & 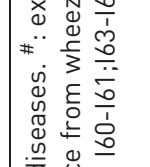 \\
\hline 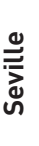 & & $\begin{array}{l}\text { 蕉 } \\
\text { 荟 } \\
\text { 怘 }\end{array}$ & $\stackrel{\circ}{\stackrel{m}{~}}$ & 六 & 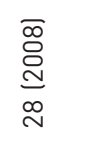 & & 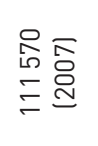 & $\check{\pi}$ & $\stackrel{\sim}{\sim}$ & 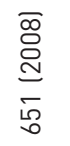 & 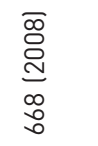 & 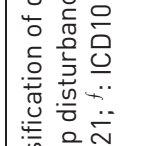 \\
\hline 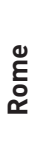 & & 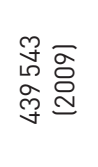 & 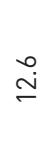 & $\underset{\text { }}{\stackrel{\sim}{j}}$ & 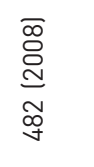 & & $\begin{array}{l}\text { o } \\
\infty \\
\infty \\
\hat{\alpha} \\
i \\
i\end{array}$ & $\bar{\therefore}$ & $\stackrel{\sim}{\sim}$ & 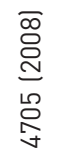 & 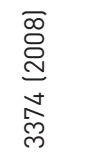 & 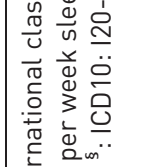 \\
\hline 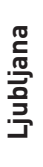 & & 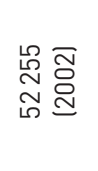 & $\stackrel{\sim}{\stackrel{\sim}{\sim}}$ & $\underset{\sim}{\stackrel{N}{\sim}}$ & 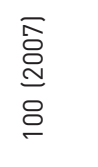 & & 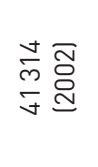 & $\bar{\therefore}$ & $\stackrel{\sim}{\sim}$ & $\begin{array}{l}\text { 令 } \\
\stackrel{d}{0} \\
a \\
o \\
j\end{array}$ & 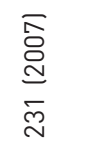 & 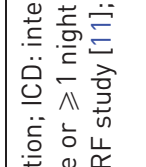 \\
\hline $\begin{array}{l}\frac{\pi}{0} \\
\frac{\pi}{0} \\
\frac{\pi}{\pi} \\
\frac{\pi}{0}\end{array}$ & & 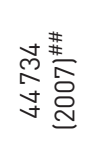 & $\stackrel{\circ}{\underset{\sim}{\sim}}$ & $\overline{\vec{m}}$ & 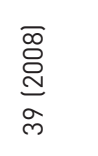 & & 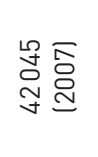 & $\check{\pi}$ & $\stackrel{\sim}{\sim}$ & 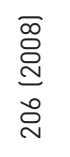 & $\begin{array}{l}\infty \\
\infty \\
\stackrel{0}{N} \\
\stackrel{\circ}{\circ}\end{array}$ & 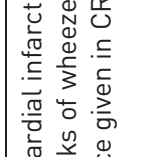 \\
\hline 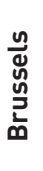 & & $\begin{array}{l}\text { Õ } \\
\text { 令 } \\
\text { స̃ }\end{array}$ & $\stackrel{m}{\sim}$ & $\stackrel{\sim}{\stackrel{m}{m}}$ & $\begin{array}{l}\bar{\Xi} \\
\stackrel{0}{\mathrm{~d}} \\
\text { 足 }\end{array}$ & & 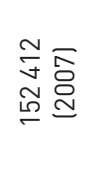 & 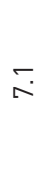 & $\stackrel{\sim}{\sim}$ & $\begin{array}{l}\overline{0} \\
\stackrel{0}{0} \\
\stackrel{0}{\infty}\end{array}$ & $\begin{array}{l}\overline{0} \\
\stackrel{\circ}{N} \\
\stackrel{N}{N} \\
\stackrel{N}{\sim}\end{array}$ & 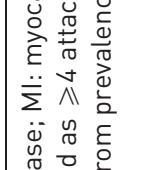 \\
\hline 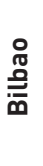 & & 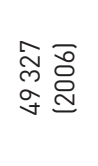 & $\stackrel{m}{\sim}$ & $\begin{array}{l}\stackrel{\circ}{\text { m }} \\
\text { 品 }\end{array}$ & 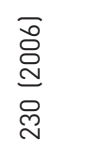 & & $\begin{array}{l}\stackrel{m}{=} \bar{\circ} \\
\stackrel{0}{\circ}\end{array}$ & 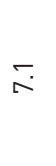 & $\stackrel{\llcorner}{\sim}$ & 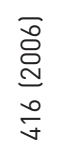 & $\begin{array}{l}\bar{\sigma} \\
\stackrel{0}{0} \\
\infty \\
\infty \\
i n\end{array}$ & 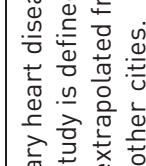 \\
\hline 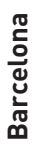 & & 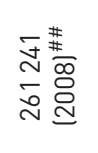 & $\stackrel{\infty}{=}$ & $\stackrel{\circ}{\stackrel{\circ}{\sim}}$ & 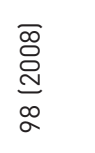 & & 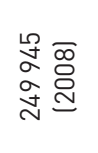 & $\bar{\therefore}$ & 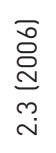 & $\begin{array}{l}\bar{\infty} \\
\stackrel{0}{0} \\
\stackrel{0}{\circ} \\
\stackrel{0}{ }\end{array}$ & 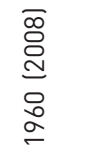 & 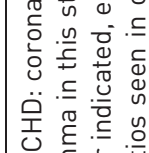 \\
\hline 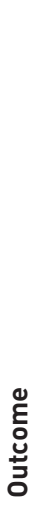 & 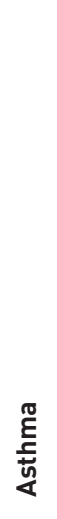 & 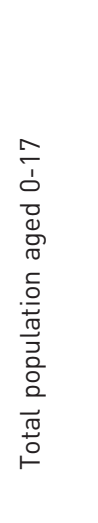 & 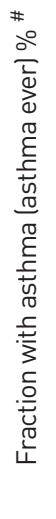 & 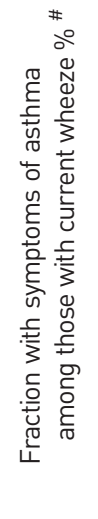 & 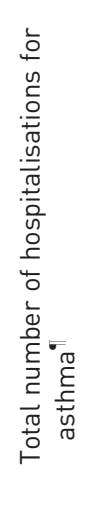 & 몽 & 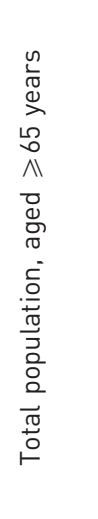 & 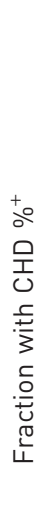 & 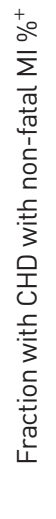 & 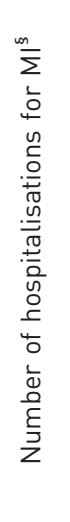 & 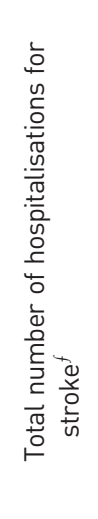 & 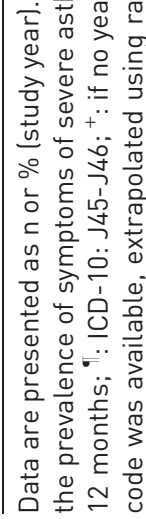 \\
\hline
\end{tabular}


cities except Stockholm, thus, we indicate the impact of further air quality improvement to levels that are thought to be realistic, as exemplified in case of Stockholm. Previous assessments used reference values as low as $7.5 \mu \mathrm{g} \cdot \mathrm{m}^{-3}$. To derive acute effects, we expressed a change in scenario in terms of the change in the annual mean concentration. Summing up daily effects due to daily changes in concentrations would have given the same results, but changes in annual means are more meaningful, especially from a policy perspective.

To reflect statistical uncertainty, we provided a point estimate and an upper and lower bound for the 95\% confidence interval of the chronic and acute CRFs combined, and $20 \%$ variability for chronic health outcomes. We used Monte Carlo simulations to propagate uncertainty distributions and to evaluate the sensitivity of our results. All calculations of disease burden were conducted at both the city level and aggregated across the 10 cities.

\section{Results}

Table 4 summarises exposure data obtained from the 10 cities and used to evaluate the burden of childhood asthma and CHD. On average, for the 10 cities considered, $31 \%$ of the combined population was estimated to live within $75 \mathrm{~m}$ of a busy road and $53 \%$ within $150 \mathrm{~m}$ of such roads. Across the 10 cities, the annual weighted mean for PM10 was $30 \mu \mathrm{g} \cdot \mathrm{m}^{-3}$ and $39 \mu \mathrm{g} \cdot \mathrm{m}^{-3}$ for $\mathrm{NO}_{2}$.

Table 5 presents the number and proportion of chronic disease cases attributable to near-road traffic-related pollution. We estimated that an average of 33200 asthma cases (14\% of all asthmatic children, with a $95 \%$ confidence interval estimated between $3 \%$ and $25 \%$ ) were attributable to near-road traffic-related pollutants. In other words, these cases would not have occurred if no one lived within the buffer zone or if those pollutants did not exist. For CHD estimates, city-specific data was not available, thus using published prevalence we estimated an average of $37200 \mathrm{CHD}$ cases were attributable to near-roadway traffic-related pollution ( $28 \%$ of all older adults with CHD, with a $95 \%$ confidence interval estimated between $9 \%$ and $44 \%$ ).

Table 6 presents the total number and percentage of selected exacerbation outcomes for childhood asthma attributable to air pollution on a yearly basis. Without considering the disease progressions induced by near-road traffic-related pollution, we estimated that reducing air pollution levels to the scenario reference values would prevent 1585 episodes of asthma symptoms (1.7\%) and 20 asthma hospitalisations (1.1\%) (table 6). The estimated preventable burden substantially increases when taking into account the notion that living near busy roads, used as a marker for near-road traffic-related pollutants, caused the development of chronic pathologies. Considering disease progressions, we estimated a total of 14400 episodes of asthma symptoms and 260 asthma hospitalisations (table 6) attributable to air pollution, accounting for $15 \%$ of acute events.

We found similar patterns for acute cardiovascular outcomes (table 7). For example, aggregated over the 10 cities, we estimated that $27 \%$ of non-fatal myocardial infarctions, $28 \%$ of myocardial infarction hospitalisations, and $26 \%$ of stroke hospitalisations were attributable to causes other than air pollution

TABLE 4 City-specific summaries of population distribution in two distance based buffers around busy roads li.e. $>10$ 000 vehicles per dayl and annual mean concentrations of $\mathrm{PM} 10$ and $\mathrm{NO}_{2}$ at urban background stations

\begin{tabular}{|c|c|c|c|c|c|c|c|c|c|c|c|}
\hline Indicator & Barcelona & Bilbao & Brussels & Granada & Ljubljana & Rome & Seville & Stockholm" & Valencia & Vienna & 10 cities $^{\circ}$ \\
\hline \multicolumn{12}{|l|}{ Traffic proximity $(\%)$} \\
\hline Year & 2007 & 2006 & 2002 & 2008 & 2006 & 2008 & 2004 & 2004 & $2007-2008$ & 2006 & \\
\hline$\leqslant 150 \mathrm{~m}$ & $77 \%$ & $59 \%$ & $64 \%$ & $28 \%$ & $47 \%$ & $43 \%$ & $38 \%$ & $30 \%$ & $71 \%$ & $62 \%$ & $53 \%$ \\
\hline \multicolumn{12}{|l|}{$\begin{array}{l}\text { Urban background } \\
\text { pollution }\end{array}$} \\
\hline $\begin{array}{l}\mathrm{NO}_{2} \text { annual } \\
\quad \text { average } \mu \mathrm{g} \cdot \mathrm{m}^{-3}\end{array}$ & 36 & 29 & 38 & 31 & 28 & 61 & 29 & 13 & 51 & 32 & 39.4 \\
\hline
\end{tabular}

PM10: particulate matter up to $10 \mu \mathrm{m}$. \#: was developed for the population of Stockholm city only (population of 790000 ) while burden is for Stockholm greater area (population of 1300000$)$; ": population weighted concentrations. 
TABLE 5 Estimated cases and per cent of lifetime childhood asthma and prevalent coronary heart disease in older adults attributable to near-road traffic-related pollution for 10 cities in Europe ${ }^{\#}$

City

Asthma (0-17 years)

\begin{tabular}{ccc}
\hline $\begin{array}{c}\text { Estimated with } \\
\text { chronic outcome } \\
n\end{array}$ & $\begin{array}{c}\text { Attributed to near-road traffic-related } \\
\text { pollution }\end{array}$ \\
\cline { 2 - 3 } & $\begin{array}{c}\text { Prevalent cases } \\
\mathrm{n}(95 \% \mathrm{Cl})\end{array}$ & $\begin{array}{c}\text { Prevalent } \\
\text { fraction } \%(95 \% \mathrm{Cl})\end{array}$
\end{tabular}

Coronary heart disease $(\geqslant 65$ years)

\begin{tabular}{|c|c|c|c|c|c|c|}
\hline & \multirow{2}{*}{$\begin{array}{l}\text { Estimated with } \\
\text { chronic outcome } \\
\text { n }\end{array}$} & \multicolumn{2}{|c|}{$\begin{array}{c}\text { Attributed to near-road traffic-related } \\
\text { pollution }\end{array}$} & \multirow{2}{*}{$\begin{array}{c}\text { Estimated with } \\
\text { chronic outcome } \\
\text { n }\end{array}$} & \multicolumn{2}{|c|}{$\begin{array}{c}\text { Attributed to near-road traffic-related } \\
\text { pollution }\end{array}$} \\
\hline & & $\begin{array}{l}\text { Prevalent cases } \\
\text { n (95\% Cl) }\end{array}$ & $\begin{array}{c}\text { Prevalent } \\
\text { fraction } \%(95 \% \mathrm{Cl})\end{array}$ & & $\begin{array}{l}\text { Prevalent cases } \\
\text { n }(95 \% \mathrm{Cl})\end{array}$ & $\begin{array}{c}\text { Prevalent } \\
\text { fraction } \%(95 \% \mathrm{Cl})\end{array}$ \\
\hline Barcelona & 30690 & $6900(1400-11500)$ & $23(5-38)$ & 17740 & 6400 (2200-9000) & $36(13-54)$ \\
\hline Bilbao & 10500 & $1200(200-2100)$ & $12(2-20)$ & 5400 & $1600(500-1500)$ & $31(10-47)$ \\
\hline Ljubljana & 15250 & $1200(200-2100)$ & $8(2-14)$ & 2930 & $700(200-1000)$ & $26(8-42)$ \\
\hline Rome & 57360 & $5700(1000-10700)$ & $10(2-19)$ & 42440 & $10300(3200-8600)$ & $24(8-40)$ \\
\hline Seville & 17680 & $1600(200-3000)$ & $9(2-17)$ & 7920 & $1700(500-2600)$ & $22(7-37)$ \\
\hline Stockholm & 24640 & $1700(300-3400)$ & $7(1-14)$ & 13010 & $2300(700-3600)$ & $18(5-31)$ \\
\hline Valencia & 12810 & $2400(400-4100)$ & $19(4-33)$ & 9170 & $3100(1100-3800)$ & $35(12-52)$ \\
\hline Vienna & 16890 & $2800(500-5200)$ & $17(3-31)$ & 19160 & $6000(2000-9200)$ & $31(11-48)$ \\
\hline
\end{tabular}

\#: truncated to zero, when negative values obtained. Estimates rounded to lowest 10th, 1000, or 10000 . ": distance to busy road depends on outcome: within $75 \mathrm{~m}$ for asthma, within $150 \mathrm{~m}$ for coronary heart disease.

among those who suffer CHD due to near-road traffic-related pollution (table 6). These cases had been previously unaccounted for.

\section{Discussion}

Our health risk assessment explored the morbidity attributable to near-road traffic-related air pollution in 10 European cities, taking into account air pollution's dual role as a cause of chronic diseases and a trigger for associated acute events. Results indicate that near-road traffic-related pollution may be responsible for a significant portion of the burden of asthma in children, as well as of CHD in older adults.

Spatial analysis showed that approximately $31 \%$ of the population live within $75 \mathrm{~m}$ of busy roads, and $53 \%$ within $150 \mathrm{~m}$. This distribution is consistent with results from other epidemiological studies in Europe [11, 35]. Notably, however, this distribution is very different from urban areas in the USA, such as Long Beach and Riverside, where a lower percentage of the population lives in close proximity to busy roads $[20,36]$. This suggests that, in Europe, exposure to and impacts of near-road traffic-related pollution may be substantial. Indeed, in cities where background pollution levels are low and further mitigation strategies may appear to be unnecessary, near-road traffic-related pollution may in fact contribute greatly to the local disease burden. For example, in Stockholm, $\mathrm{PM} 10$ and $\mathrm{NO}_{2}$ levels were below our reference values and the WHO recommended standards, yet approximately $7 \%$ of asthma cases and associated acute events were attributable to near-road traffic-related pollution.

For the acute events selected, we found that the burden attributable to near-road traffic-related pollution was on average 30 times larger than estimates which did not consider exposure both as a cause of underlying chronic disease as well as a trigger for acute events. These findings indicate that reducing near-road trafficrelated pollution would be an effective way to reduce the health burden and economic cost of both chronic disease and acute events [37].

Several studies in high income countries estimate that as much as $50 \%$ of the reduction in deaths from coronary diseases over the past 20 years can be attributed to preventive measures, such as lowering cholesterol levels, smoking prevalence, blood pressure, and physical inactivity $[38,39]$. Our estimates for chronic diseases attributable to living near busy roads are quite large. Thus, assuming a causal relationship between near-road traffic-related pollution and CHD, these results suggest that decreasing pollution near roads could be an effective additional preventive measure in the long term.

Our approach is based on two major assumptions and related uncertainties: 1) that near-road traffic-related pollution plays a part in the development of chronic diseases; and 2) that urban background air pollution 


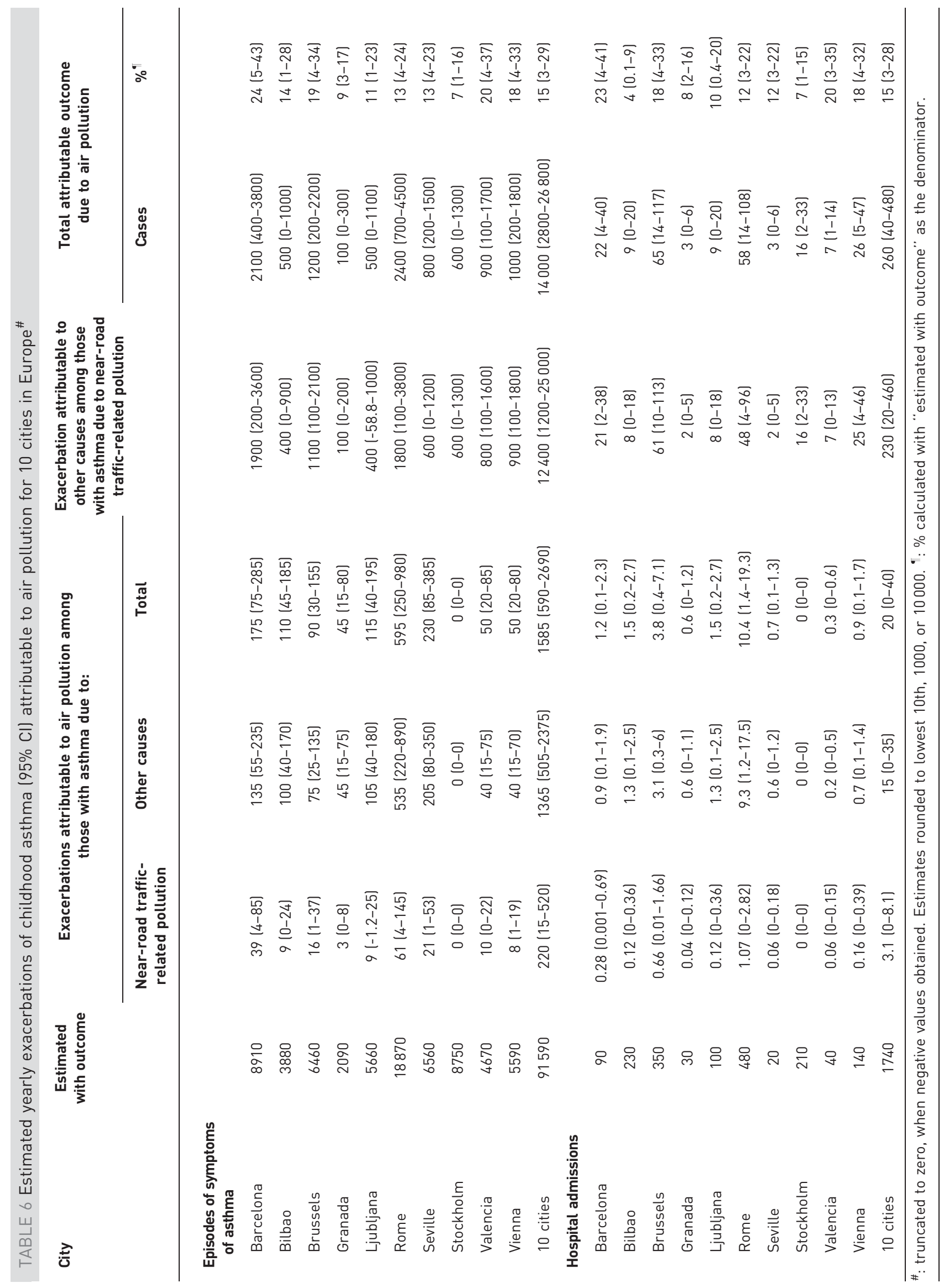


exacerbates these diseases. The degree and type of uncertainty related to these assumptions differ, depending on the outcome studied.

In the case of asthma, the role that air pollution plays in exacerbating symptoms has been established for a long time, while the role that air pollution plays in asthma onset in children has only emerged in recent years [3, 4, 40]. The Health Effect Institute (HEI) classified the evidence that long-term exposure to near-road traffic-related pollutants may lead to asthma onset as "sufficient evidence" or "suggestive but not sufficient" [5]. A highly relevant analysis of the Children's Health Study, a cohort study of school age children, identified an interaction between genetic factors and the risk of asthma incidence among those living close to highways, a finding that is very hard to explain with uncontrolled biases or confounding factors [41]. However, there are additional uncertainties related to this outcome. The question remains whether removal of near-road pollution would in fact prevent or only delay the onset of the chronic underlying pathologies [3]. In the latter case, the burden will be overestimated; however, delaying the onset of asthma symptoms even for a few years may represent a large improvement in quality of life and savings for families [37]. Furthermore, the Dutch birth cohort study followed children up until 8 years old, and found no evidence supporting postponed onset, validating our assumption [42].

In the case of myocardial infarction, there is also a large amount of evidence that acute events are related to high daily levels of air pollution $[5,43,44]$. The evidence for a link between pollution and underlying chronic conditions is less established, as there are few population studies of near-road pollutants or proximity for these outcomes. However, the evidence that atherosclerosis is related to particulate matter levels is very strong in animal studies, and at least suggestive in some epidemiological studies in humans [18]. There is also considerable biological evidence of mechanistic pathways linking air pollution to myocardial infarction, such as oxidative stress and inflammation [43]. Considering these factors, the HEI classified the evidence for the association between traffic pollution and cardiovascular disease as "suggestive but not sufficient". Given current knowledge, the extent to which near-roadway traffic pollution contributes to the progression of atherosclerosis to the point of increasing the individual's risk of myocardial infarction is unknown, and selecting a pool of individuals at risk of developing acute symptoms remains a challenge.

The interpretation of PAFs has several limitations. For most complex diseases, morbidity develops because of the interaction of several risk factors. This multi-causal model implies that it is sufficient to eliminate or remove any one of the factors contributing to the disease in order to prevent it [45]. PAF are generally developed one risk factor at a time, ignoring the potential effects of interactions between contributing factors. If one sums the PAFs derived separately for each potential risk factor for a specific disease, the total PAF for the disease may add up to more than $100 \%$ (greater than the total number of cases) since attributable cases for individuals with more than one contributing factor may have been counted more than once $[46,47]$. We present our results assuming that one risk factor is eliminated, with all other risk factors remaining unchanged. The large PAFs of disease prevalence due to near road traffic exposure is comparable to what has been found for other preventable risk factors, such as smoking. This is driven primarily by the very large fraction of the population living under conditions of high exposure, and underscores the substantial relevance of this exposure to public health. Near-road traffic-related pollution attributable fraction does represent a preventable risk factor.

Nonetheless, because of the complex chain of biological and behavioural interactive pathways at play and the uncertainty in the lag time of health changes that may result from small stepwise reductions in nearroad traffic-related pollutants, we cannot predict the true benefits of reducing near-road traffic-related exposure. If causality is true, our results indicate that decreasing exposure to near-road traffic-related pollution could effectively contribute to prevention of some chronic diseases. The extent to which reduced exposure will decrease the disease burden, however, remains unknown, and depends on changes in other risk factors. For example, in a real world scenario, the adverse effects of an increasing obesity epidemic may outweigh the benefits of reducing near-road traffic-related pollution, thus, disease rates may increase despite improvements in air quality.

For the two chronic outcomes evaluated in our study, we used the CRF of a single rather than a pooled estimate. This is a major limitation of the assessment, as we do not know how representative the CRFs are for the European cities of our study. We could not identify a sufficient number of studies that would allow for the derivation of a meta-analytical estimate, because the available studies differ largely in their approach to defining exposure metrics (e.g. chosen cut-off of distance and type of roads selected). While living close to busy roads was positively associated with asthma prevalence in children from both American [48-50] and European studies [12, 16, 51-53] the local urban layout, meteorology, fuel type, vehicle fleet or population susceptibility may differ, and potentially influence the exposure-response 


\section{TABLE 7 Estimated yearly exacerbations of coronary heart disease attributable to air pollution for 10 cities in Europe ${ }^{\#}$}

\section{Exacerbations}

\section{Estimated} exacerbations
Exacerbations attributable to air pollution among those with coronary heart disease due to:
Exacerbations attributable to other causes among those with coronary heart disease due to near-road traffic-related pollution
Total attributable exacerbations due to air pollution

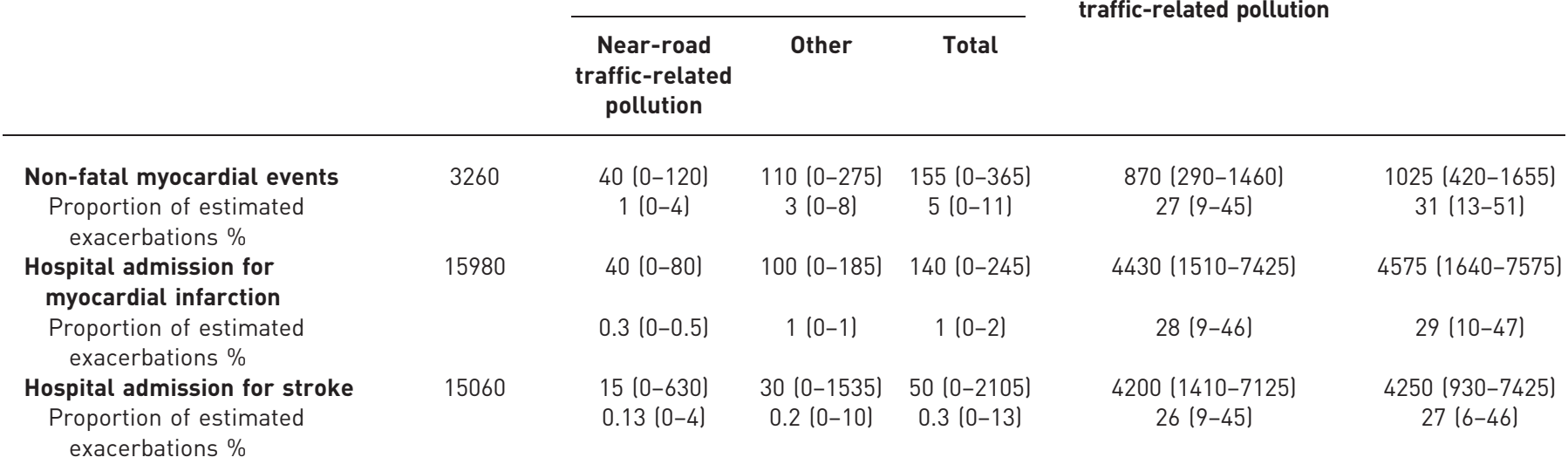

Data are presented as $\mathrm{n}, \mathrm{n}(95 \% \mathrm{Cl})$ or $\%(95 \% \mathrm{Cl})$ of the number of estimated exacerbations. ${ }^{*}$ : truncated to zero, when negative values obtained. Estimates rounded to nearest multiplier.

relationship. Thus, extrapolation across regions for near-roadway traffic-related exposure is particularly delicate. Considering the lack of data, and to remain consistent with our previous methodological papers, we chose to keep the MCCONNELl et al. [20] 2006 risk estimate in our analysis, derived for Southern Californian children. In the USA, in particular the Californian communities studied previously, street canyons are far less common, and the distance from traffic lanes to homes is generally much larger than in typical European cities. Near-road traffic-related pollution levels are likely higher in Europe relative to the USA, and our use of methodology developed in the USA may underestimate the health impact in Europe.

Moreover, we did not consider the impact of other chronic and acute outcomes also potentially associated with near-road traffic-related pollution and/or regional urban pollution, such as asthma in adults, chronic pulmonary obstructive disease, cognitive development in children or reproductive outcomes [5, 54]. Asthma is most likely under-diagnosed, thus the prevalence used to derive attributable cases may be too low [33]. We may have ignored population exposure to traffic pollutants along smaller streets which might also have high levels of traffic-related pollutants, particularly diesel emissions, in European cities where street canyons prevail, such as Barcelona. Therefore, we believe that our approach underestimates the health impact of near-road traffic-related pollution in Europe.

Another limitation of our study is related to using traffic proximity as a proxy for exposure to traffic-related pollution. One perspective suggests that such an approach lacks proper adjustments for socioeconomic factors or other indirect confounders associated with proximity to busy roads, resulting in biased estimates. A contrasting perspective holds that distance-outcome risk functions are less biased than others, because they better adjust for non-measurable confounders within the exposure term [5].

We used residential proximity to traffic to represent long-term exposure to near-road traffic-related pollution because this metric is applicable to impact analyses across several cities. Studies do show association between chronic diseases and small-scale apportioned traffic-related pollutants (such as $\mathrm{NO}_{2}$, PM2.5 and soot) $[5,55]$; however, in order to incorporate this data into impact assessments, more detailed information is needed concerning the spatial distribution of pollutants. Such data is not yet available for a large number of cities. In contrast, the spatial distribution of people and traffic can be studied relatively easily and cheaply. Therefore, using residential proximity to traffic to represent long-term exposure to nearroad traffic-related pollution represents an effective practical approach.

Nonetheless, our choice of proxy does not reflect the variability of the concentration and toxicity of pollution that may exist within and across cities, due to differences in meteorology, type of fleet, street canyon effects and predominant fuel use [5]. For example, we emphasise that traffic emissions are a major source of the pollutants used in our model for urban background pollution; however, the fraction 
attributable to traffic varies across cities. More relevant scenarios could also be explored if more data related to CRFs and spatial models of source specific pollutants was available.

In our analysis, the level of detail available to develop exposure distributions and other input data varied across cities. Our sensitivity analysis showed that the large confidence interval around the chronic CRFs drives most of the uncertainty around the point estimates (results not shown), nonetheless, our experience calls for better integration of future environmental and health monitoring systems to obtain health assessments with fewer uncertainties.

In conclusion, we believe that it is important to move beyond traditional health impact assessments, largely focused on mortality, and expand our approach to include the potential health impacts of near-road trafficrelated pollution on morbidity. Despite uncertainty and limitations, our results indicate that near-road traffic-related pollution may be responsible for a large but preventable burden of chronic diseases and related acute morbidities in urban areas.

\section{Acknowledgements}

The huge amount of work behind the APHEKOM project is the fruit of the generous and constructive input from all the members of the APHEKOM network and in particular to all our local city partners. For this study, we would like to additionally acknowledge Marisa Estarlich (Center for Public Health Research (CSISP), Valencia and Spanish Consortium for Research on Epidemiology and Public Health (CIBERESP)), Teresa Martín (Dirección Territorial de Sanidad de Bizkaia, Bilbao, Basque Country, Spain) and Natalia Valero ( Agencia de Salut Publica de Barcelona). We thank Amena Briét (Swiss Tropical and Public Health Institute) and Bruno Schull (Basel, Switzerland) for revising the English syntax and editing the manuscript.

\section{References}

1 Künzli N, Kaiser R, Medina S, et al. Public-health impact of outdoor and traffic-related air pollution: a European assessment. Lancet 2000; 356: 795-801.

2 Ballester F, Medina S, Boldo E, et al. Reducing ambient levels of fine particulates could substantially improve health: a mortality impact assessment for 26 European cities. J Epidemiol Community Health 2008; 62: 98-105.

3 Anderson HR, Favarato G, Atkinson RW. Long-term exposure to air pollution and the incidence of asthma: metaanalysis of cohort studies. Air Qual Atmos Health 2013; 6: 47-56.

4 Anderson HR, Favarato G, Atkinson RW. Long-term exposure to outdoor air pollution and the prevalence of asthma: meta-analysis of multi-community prevalence studies. Air Qual Atmos Health 2013; 6: 57-68.

5 HEI. Panel on the Health Effects of Traffic-Related Air Pollution. Traffic-related Air Pollution: A Critical Review of the Literature on Emissions, Exposure, and Health Effects. HEI Special Report 17. Boston, Health Effects Institute, 2010.

6 Commission of the European Communities. Commission Staff Working Paper. Annex to: The Communication on Thematic Strategy on Air Pollution and The Directive on "Ambient Air Quality and Cleaner Air for Europe. Impact assessment. 2005. http://ec.europa.eu/environment/archives/cafe/pdf/ia_report_en050921_final.pdf

7 US Environmental Protection Agency. Regulatory Impact Analysis. 2006 National Ambient Air Quality Standards for Particle Pollution. www.epa.gov/ttnecasl/ria.html Date last accessed: July 20, 2009.

8 Cho SH, Tong H, McGee JK, et al. Comparative toxicity of size-fractionated airborne particulate matter collected at different distances from an urban highway. Environ Health Perspect 2009; 117: 1682-1689.

9 Hazenkamp-von Arx ME, Schindler C, Ragettli MS, et al. Impacts of highway traffic exhaust in alpine valleys on the respiratory health in adults: a cross-sectional study. Environ Health 2011; 10: 13.

10 Hoffmann B, Moebus S, Mohlenkamp S, et al. Residential exposure to traffic is associated with coronary atherosclerosis. Circulation 2007; 116: 489-496.

11 Hoffmann B, Moebus S, Stang A, et al. Residence close to high traffic and prevalence of coronary heart disease. Eur Heart J 2006; 27: 2696-2702.

12 Janssen NA, Brunekreef B, van Vliet P, et al. The relationship between air pollution from heavy traffic and allergic sensitization, bronchial hyperresponsiveness, and respiratory symptoms in dutch schoolchildren. Environ Health Perspect 2003; 111: 1512-1518.

13 Modig L, Jarvholm B, Ronnmark E, et al. Vehicle exhaust exposure in an incident case-control study of adult asthma. Eur Respir J 2006; 28: 75-81.

14 Morgenstern V, Zutavern A, Cyrys J, et al. Respiratory health and individual estimated exposure to traffic-related air pollutants in a cohort of young children. Occup Environ Med 2007; 64: 8-16.

15 Tonne C, Melly S, Mittleman M, et al. A case-control analysis of exposure to traffic and acute myocardial infarction. Environ Health Perspect 2007; 115: 53-57.

16 Wjst M, Reitmeir P, Dold S, et al. Road traffic and adverse effects on respiratory health in children. BMJ 1993; 307: 596-600.

17 Kunzli N, Perez L, Lurmann F, et al. An attributable risk model for exposures assumed to cause both chronic disease and its exacerbations. Epidemiology 2008; 19: 179-185.

18 Kunzli N, Perez L, von Klot S, et al. Investigating air pollution and atherosclerosis in humans: concepts and outlook. Prog Cardiovasc Dis 2011; 53: 334-343.

19 Perez L, Künzli N, Avol E, et al. Global goods movement and the local burden of childhood asthma in Southern California. Am J Public Health 2009; 99: Suppl. 3, 5622-5628.

20 McConnell R, Berhane K, Yao L, et al. Traffic, susceptibility, and childhood asthma. Environ Health Perspect 2006; 114: 766-772.

21 Weinmayr G, Romeo E, De Sario M, et al. Short-term effects of $\mathrm{PM}_{10}$ and $\mathrm{NO}_{2}$ on respiratory health among children with asthma or asthma-like symptoms: a systematic review and meta-analysis. Environ Health Perspect 2010; 118: 449-457. 
22 Andersen ZJ, Wahlin P, Raaschou-Nielsen O, et al. Size distribution and total number concentration of ultrafine and accumulation mode particles and hospital admissions in children and the elderly in Copenhagen, Denmark. Occup Environ Med 2008; 65: 458-466.

23 Atkinson RW, Anderson HR, Sunyer J, et al. Acute effects of particulate air pollution on respiratory admissions: results from APHEA 2 project. Air pollution and health: a European approach. Am J Respir Crit Care Med 2001; 164: $1860-1866$.

24 Zhang J, Yu KF. What's the relative risk? A method of correcting the odds ratio in cohort studies of common outcomes. JAMA 1998; 280: 1690-1691.

25 Peters A, von Klot S, Heier M, et al. Particulate air pollution and nonfatal cardiac events. Part I. Air pollution, personal activities, and onset of myocardial infarction in a case-crossover study. Res Rep Health Eff Inst 2005; 1-66.

26 D'Ippoliti D, Forastiere F, Ancona C, et al. Air pollution and myocardial infarction in Rome: a case-crossover analysis. Epidemiology 2003; 14: 528-535.

27 Lanki T, Pekkanen J, Aalto P, et al. Associations of traffic related air pollutants with hospitalisation for first acute myocardial infarction: the HEAPSS study. Occup Environ Med 2006; 63: 844-851.

28 Poloniecki JD, Atkinson RW, de Leon AP, et al. Daily time series for cardiovascular hospital admissions and previous day's air pollution in London, UK. Occup Environ Med 1997; 54: 535-540.

29 Halonen JI, Lanki T, Yli-Tuomi T, et al. Particulate air pollution and acute cardiorespiratory hospital admissions and mortality among the elderly. Epidemiology 2009; 20: 143-153.

30 Larrieu S, Jusot JF, Blanchard M, et al. Short term effects of air pollution on hospitalizations for cardiovascular diseases in eight French cities: the PSAS program. Sci Total Environ 2007; 387: 105-112.

31 Le Tertre A, Medina S, Samoli E, et al. Short-term effects of particulate air pollution on cardiovascular diseases in eight European cities. J Epidemiol Community Health 2002; 56: 773-779.

32 Oudin A, Stromberg U, Jakobsson K, et al. Estimation of short-term effects of air pollution on stroke hospital admissions in southern Sweden. Neuroepidemiology 2010; 34: 131-142.

33 Lai CK, Beasley R, Crane J, et al. Global variation in the prevalence and severity of asthma symptoms: phase three of the international study of asthma and allergies in childhood (ISAAC). Thorax 2009; 64: 476-483.

34 World Health Organization. WHO air quality guidelines for particulate matter, ozone, nitrogen dioxide and sulfur dioxide. Global update 2005. Summary of risk assessment. Geneva, WHO Press, 2005. Available at: http:// whqlibdoc.who.int/hq/2006/WHO_SDE_PHE_OEH_06.02_eng.pdf

35 Schikowski T, Sugiri D, Ranft U, et al. Long-term air pollution exposure and living close to busy roads are associated with COPD in women. Respir Res 2005; 6: 152.

36 Rioux CL, Gute DM, Brugge D, et al. Characterizing urban traffic exposures using transportation planning tools: an illustrated methodology for health researchers. J Urban Health 2010; 87: 167-188.

37 Brandt SJ, Perez L, Künzli N, et al. Costs of childhood asthma due to traffic-related pollution in two California communities. Eur Respir J 2012; 40: 363-370.

38 Ford ES, Ajani UA, Croft JB, et al. Explaining the decrease in U.S. deaths from coronary disease, 1980-2000. N Engl J Med 2007; 356: 2388-2398.

39 Unal B, Critchley JA, Capewell S. Explaining the decline in coronary heart disease mortality in England and Wales between 1981 and 2000. Circulation 2004; 109: 1101-1107.

40 Anderson HR, Ruggles R, Pandey KD, et al. Ambient particulate pollution and the world-wide prevalence of asthma, rhinoconjunctivitis and eczema in children: phase one of the international study of asthma and allergies in childhood (ISAAC). Occup Environ Med 2010; 67: 293-300.

41 Salam MT, Gauderman WJ, McConnell R, et al. Transforming growth factor-1 c-509t polymorphism, oxidant stress, and early-onset childhood asthma. Am J Respir Crit Care Med 2007; 176: 1192-1199.

42 Carlsten C, Dybuncio A, Becker A, et al. Traffic-related air pollution and incident asthma in a high-risk birth cohort. Occup Environ Med 2011; 68: 291-295.

43 Brook RD, Rajagopalan S, Pope CA 3rd, et al. Particulate matter air pollution and cardiovascular disease: an update to the scientific statement from the American Heart Association. Circulation 2010; 121: 2331-2378.

44 Nawrot TS, Perez L, Kunzli N, et al. Public health importance of triggers of myocardial infarction: a comparative risk assessment. Lancet 2011; 377: 732-740.

45 Rockhill B, Newman B, Weinberg C. Use and misuse of population attributable fractions. Am J Public Health 1998; 88: 15-19.

46 Levine B. What does the population attributable fraction mean? Prev Chronic Dis 2007; 4: A14.

47 Rothman KJ, Greenland S. Causation and causal inference in epidemiology. Am J Public Health 2005; 95: Suppl. 1, S144-S150.

48 Gauderman WJ, Avol E, Lurmann F, et al. Childhood asthma and exposure to traffic and nitrogen dioxide. Epidemiology 2005; 16: 737-743.

49 Gordian ME, Haneuse S, Wakefield J. An investigation of the association between traffic exposure and the diagnosis of asthma in children. J Expo Sci Environ Epidemiol 2006; 16: 49-55.

50 Kim JJ, Huen K, Adams S, et al. Residential traffic and children's respiratory health. Environ Health Perspect 2008; 116: 1274-1279.

51 Lewis SA, Antoniak M, Venn AJ, et al. Secondhand smoke, dietary fruit intake, road traffic exposures, and the prevalence of asthma: a cross-sectional study in young children. Am J Epidemiol 2005; 161: 406-411.

52 Nicolai T, Carr D, Weiland SK, et al. Urban traffic and pollutant exposure related to respiratory outcomes and atopy in a large sample of children. Eur Respir J 2003; 21: 956-963.

53 van Vliet $\mathrm{P}$, Knape M, de Hartog J, et al. Motor vehicle exhaust and chronic respiratory symptoms in children living near freeways. Environ Res 1997; 74: 122-132.

54 Guxens M, Aguilera I, Ballester F, et al. Prenatal exposure to residential air pollution and infant mental development: modulation by antioxidants and detoxification factors. Environ Health Perspect 2012; 120: 144-149.

55 Gehring U, Wijga AH, Brauer M, et al. Traffic-related air pollution and the development of asthma and allergies during the first 8 years of life. Am J Respir Crit Care Med 2010; 181: 596-603. 